\title{
TOOLS OF THE TRADE
}

\section{Measuring stream temperature using thermal infrared imagery}

Stream temperature, an indicator of water quality and in situ processes, influences in-stream nutrient cycling, dissolved oxygen and solute concentrations. The temperature is typically measured using conventional in-stream sensors such as fibre optic technology and data loggers, both of which have restricted spatial coverage. Data loggers, for instance, must be installed everywhere a measurement is needed, and fibre optic cables can measure temperatures approximately every centimetre along a stream transect but are cumbersome to deploy, especially in inaccessible locations. Given these limitations, thermal infrared (TIR) imagery has been tested as a tool to measure stream temperature.

Ground-based cameras and unmanned aerial vehicles (UAVs) are used to record the average temperature for every pixel using TIR, with pixel size being determined by distance from the stream and camera resolution. For example, a greater portion of the stream surface temperature is averaged into a single pixel when the TIR camera is positioned further from the stream.
Importantly, though, researchers must correct for stream surface emissivity and reflected environmental temperatures, as uncorrected values can be inaccurate owing to hot or cold reflections, or appear accurate only because the reflected temperatures are similar to the stream temperature. Although correction and validation require the use of data loggers, only a few are needed as control points, greatly reducing the time and equipment required for in-stream measurements and providing whole surface temperature maps.

TIR imagery produces high spatial and temporal resolution stream temperature data, providing valuable insights into stream temperature dynamics. TIR UAV surveys have revealed the shape and extent of wastewater and urban runoff thermal plumes exiting culverts. Similarly, they have identified locations of cool groundwater seeps that provide habitat for cold-water fish. Furthermore, the energy due to longwave radiation from terrain surrounding glaciers has been measured using TIR imagery, improving estimates of ablation rates and

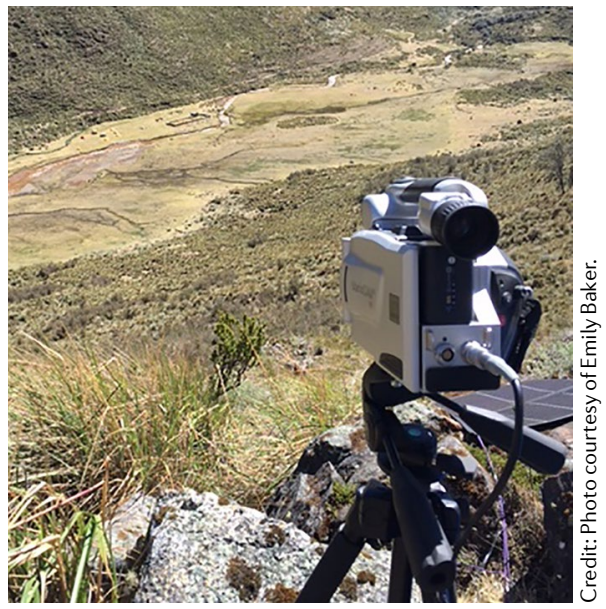

meltwater production. While correction methods are still improving, TIR imagery offers an analytical technique to measure stream temperatures remotely.

Emily A. Baker Syracuse University, Syracuse, NY, USA. e-mail: eabaker@syr.edu

The author declares no competing interests. 